\title{
Establishing Criteria of Rigor and Relevance in Interaction Design Research
}

\author{
Daniel Fallman \\ Interactive Institute Umeå \\ Umeå University, Umeå, Sweden \\ daniel.fallman@tii.se
}

\author{
Erik Stolterman \\ School of Informatics and Computing \\ Indiana University at Bloomington, Indiana, USA \\ estolter@indiana.edu
}

\begin{abstract}
Interaction design research has rapidly evolved into a unique discipline embracing practicing professionals, design educators, and academic researchers. As with many evolving disciplines, attracting attention from a large number of people with different backgrounds, interests, and ways of seeing tends to cause 'disciplinary anxiety', which inevitably leads to the question of what constitutes 'good research'. What is rigorous and relevant interaction design research? How do we recognize and evaluate it? In this paper, we argue that most current attempts at dealing with issues of rigor and relevance in interaction design research tend to be on loan from other disciplines, and tend to overlook, conceal, or knowingly exclude some of what makes interaction design research such a unique field. Our primary contribution is that what may be perceived as three different design research activities-design practice, design exploration, and design studies-have their own purposes, intended outcomes, and internal logic. Each form of research must thus be examined in its own right and the notions of rigor and relevance for each of them have to be based on a firm understanding of the particular purpose of each approach. We would argue that this is not done consistently in the field today, which leads to misunderstandings, confusion, and mistakes when interaction design research is reviewed, discussed, and assessed.
\end{abstract}

Rigor and relevance. Interaction design research. Design practice. Design Exploration. Design Studies.

\section{INTRODUCTION}

Interaction design research has rapidly evolved into a unique, thriving discipline embracing practicing professionals, design educators, and academic researchers. As with many evolving disciplines, attracting attention and effort from a large number of people with different backgrounds, interests, and ways of seeing also tends to cause what can perhaps best be described as 'disciplinary anxiety'. Where are we going, what is the core of the field, what are relevant research questions, what are appropriate methods? Questions like these inevitably lead to the more general subject: what constitutes 'good research' and how do we recognize and evaluate it? What is rigorous and relevant interaction design research?

This paper attempts to commence such a discussion by looking in some detail at the concepts of rigor and relevance.

\subsection{Disciplinary Anxiety}

Disciplinary anxiety can be experienced in a field when heterogeneous ways of doing research lead to diverse assumptions about what constitutes legitimate research. We find this to be the case in interaction design research today, where notions of legitimacy are being thrown around without being paid enough attention. Often, these notions tend implicitly or explicitly to be on loan from other disciplines, such as Human-Computer Interaction $(\mathrm{HCl})$, product design, computer science, cognitive science, anthropology, and so on.

The current situation is not surprising and can be understood as a sign of some inherent conditions in the field today. First, the disciplinary borders between the field of interaction design research and other design and art disciplines is neither entirely clear nor generally accepted and the relationship to more established fields of research-including traditional $\mathrm{HCl}$, Computersupported Collaborative Work (CSCW), Information Systems (IS), Computer Science, and Cognitive Science-is not clear.

Second, most traditional design educations have only recently started to involve themselves in academic research. In practical terms, this means that many such organizations are neither in possession of a research tradition nor experienced supervisors to build and guide students and inexperienced researchers. In addition, new Ph.D. 
students in design research are typically recruited from masters programs that tend to prepare them for practicing design, not for the practice of interaction design research.

Third, the available venues for presenting new research in interaction design, including conferences and journals, allow for substantial latitude in terms of acknowledged research methods and approaches, ranging from quantitative empirical lab studies and qualitative observational studies to research-through-design and critical design experiments. While we do not argue against the richness this brings to the field, we do however note that it has practical consequences for the research that is being carried out. Inexperienced researchers and students going into interaction design research tend to find the choice and use of research methods quite overwhelming and difficult. This is especially true in relation to methods and techniques that are not directly linked or compatible with their understanding of the design process. We therefore see a tendency among new interaction design researchers-especially those from an art and design school background-to be unsure of basic research issues, such as the difference between qualitative and quantitative research, induction and deduction, relation between claim and evidence, etc. The richness and openness provided by the field as a whole, when it comes to approaches and methods, may seem confusing to the individual who might, rather than embrace the openness, instead chose to stick to his or her own quite small research toolbox and only discuss it with likeminded. This leads to a shattered discipline with many small communities, incommensurable with each other.

Taken together, at least on the surface or as seen from another discipline, interaction design research seems to lack rigor.

A substantial amount of effort in interaction design research has been focused on developing new theoretical approaches, methods, tools, and techniques intended to support interaction designers in their practice. While this research has enriched the field with a diverse set of approaches, methods, and techniques, the success of these contributions are not unquestionable. Rogers (2004), for instance, notes that these contributions are not always useful for practitioners in that they are too time-consuming, too difficult to learn, too abstract and theoretical, or that they do not lead to desired results when actually used in practice. Stolterman (2008) argues that one reason why research aimed at supporting design practice is not always successful is that it has not been grounded in and guided by a sufficient understanding and acceptance of the nature of design practice. As a consequence, researchers have developed and/or adopted and adapted approaches and methods that may be successful in their respective research settings, but which are not always appropriate for interaction design practice.

If this is the case, then research in interaction design also appears to be lacking in relevance.

Hence, is interaction design research a field that lacks both rigor and relevance? To try to understand this question better, we will first need to look at some previous ideas of the relationship between the terms rigor and relevance, and then compare these ideas with what we see as some specific qualities or characteristics of interaction design research.

\section{RIGOR AND RELEVANCE}

While the topic of rigor and relevance has only recently surfaced as an explicit issue in interaction design research, similar discussions have a longer history in some related disciplines. For instance, the field of Information Systems (IS) has quite intensely discussed various issues of rigor and relevance for more than a decade. Robey \& Marcus (1998) note that one of the main reasons for having the discussion on rigor and relevance in the first place is the perceived need to establish the field academically while at the same time be practitioner-oriented. This is in line with the current state of interaction design research: how do we build a body of knowledge that is both credible academically as well as relevant to practicing interaction designers and society at large?

\subsection{Qualities of Rigorous Research}

When discussing quality of research in a broad sense, two characteristics tend to be emphasized: validity and reliability. Validity can be thought of as "judgments about whether you are 'measuring', or explaining, what you claim to be measuring or explaining" (Mason 1996, p. 146). Reliability typically refers to the idea that two or more researchers studying the same phenomenon would come up with compatible results.

Already at this stage, some remarks must be made. It is commonly understood that these concepts are rooted in a positivistic-quantitative research tradition. In this tradition, the concept of reliability embodies the notion of the presumed quality of the instruments with which data is collected. In other kinds of research, for instance interpretativequalitative research, the researcher has an active and thus by definition non-objective role in collecting the data and interpreting what is going on in a certain situation. There are attempts at establishing forms of validity in this tradition as well that, often misguided, draws from the same roots 
as within more positivistic and quantitative approaches. Likewise, the concept of validity traditionally promotes a certain scientific worldview that does not easily harmonize with for instance a view of science were reality is socially constructed. Again, there are attempts at establishing other forms of validity that would better serve other assumptions about reality and how to study it.

In interaction design research, seen as a design discipline, the uncertainty as to the applicability of these concepts is even greater. What would constitute the validity and reliability of a designed object or of knowledge produced by a designerly approach? For instance, it appears very unlikely that two design researchers would come up with the same outcome even in the improbable case that they are provided exactly equal settings, materials, tools, users, etc. One could even argue that a specific quality and value of design research is its 'unreliability' and 'invalidity', manifested in creativity, innovation, and different ways of seeing. Hence, in some situations, validity and reliability in its traditional sense might not even be desirable.

\subsection{Qualities of Relevant Research}

A major theme in the IS debate on rigor and relevance is a perceived lack of relevance in that practicing IS professionals do not appear to be very interested in the field's findings. Keen (1991) suggests that in order to be relevant, research has to be interesting, applicable, current, and accessible. Interesting research addresses problems, challenges, or themes that are important to professionals. If practitioners can utilize the results and findings-often in the form of new knowledge and/or methods and techniques-we might call them applicable. Research that addresses timely issues that practitioners deal with at the moment can be seen as a sign of one's research being current. Finally, research is accessible if it is presented in an understandable way to the practitioners.

What is considered relevant research also tends to make a substantial contribution, and in order to do so the research often has to be original or new. Also, the potential for generalizability of one's research findings typically helps in establishing relevance too, since it means that the findings will cover a broader scope of situations and applications. In interaction design research, it is however not uncommon that work is presented as a 'single case' where the authors stress the disclaimer that they make no claim for the generalizability of the study. What is often implicitly meant by generalizations in such cases is empirical generalization, where findings from the studied sample population are extended to a wider group of people based on the argument that the sample in some sense is representative of the wider population. However, other generalizations, such as theoretical generalizations, extend findings to theoretical propositions rather than to populations (see Yin 1994; Lee and Baskerville, 2003). Even though there are some recent examples (e.g. Stolterman \& Wiberg, 2010), such generalizations are very rarely discussed in relation to interaction design research.

\section{THREE FORMS OF INTERACTION DESIGN RESEARCH?}

We argue that most current attempts at dealing with issues of rigor and relevance in interaction design research tend to be on loan from other disciplines, and may thus overlook, conceal, or knowingly exclude some of what makes interaction design research such a unique field. While there have been many attempts at capturing the special characteristics of design research (see for instance Buchanan 1996, Cross, 1999, Roth, 1999, Fallman, 2003, Zimmerman et al., 2007; Stolterman, 2008), we use Fallman's (2008) triangular model of design research as a starting point to help us define a number of key concepts and some basic conditions and tensions between what we see as different kinds of interaction design research activities. We do this based on the assumption, which also becomes the general contribution of this paper, that each design research activity has its own purpose and intended outcome and that rigor and relevance have to be defined and measured in relation to what the intention and outcome of the activity is.

\subsection{The Interaction Design Research Triangle}

Fallman's (2008) model is a triangle that presents a two-dimensional space for plotting the position of a design research activity drawn up in between three extremes: design practice, design studies, and design exploration.

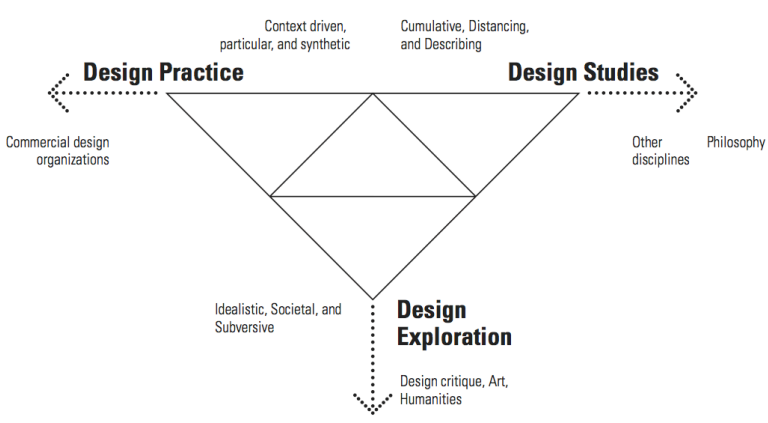

Figure 1: The interaction design triangle

A key concept of the model is that the actual methods, techniques, and tools used in the different activity areas can be quite similar. Rather, they are primarily different in perspective, purpose, 
and tradition. Before we further examine the role of rigor and relevance in interaction design research we will elaborate briefly on each of the three forms of research as presented in the triangle model and initiate some questions concerning rigor and relevance.

\subsection{Research as Design Practice}

Design Practice is the kind of activities that interaction design researchers are involved with that are very close, if not identical, to the kinds of activities carried out by practicing interaction designers. Here, interaction design researchers should be part of a design team as first and foremost a designer, not as an outside observer 'participating' in design. The area is primarily synthetic to its character; it involves and engages the researcher in a particular design situation with the ultimate purpose of transforming an unwanted situation into a preferred one through design. This calls for a certain level of participation and commitment on the researcher's part, i.e. involvement in a design team and a strong commitment to help build successful products and services. This type of purpose and commitment has several implications for how we can understand rigor and relevance.

In this kind of research, the design researcher takes a proactive part in the process. In doing so, the design researcher also typically comes to be part of a real design team whose members have different skills and different worldviews. For such a team to work, they need to find ways of sharing meaning across disciplinary boundaries, which means that what is considered core competence is not a given and the researcher is just one of many individuals bringing competence to the team. In design practice projects, the concept of a client is strong and there is most often a contractual relationship between the designers and the client that govern the direction of the process. A design practice project typically takes place in some sort of field setting that, especially if the project runs over some length of time, will come to confront issues of relevance.

Overall, this form of design research is hence strongly shaped by the practical concern of designing and developing a practical and usable design for a particular setting and client with a particular purpose.

\subsection{Research as Design Explorations}

Design Exploration has a synthetic and proactive character; the interaction design researcher is typically involved in bringing forth an artifact of some kind. But rather than user needs, client demands or market opportunities, design explorations extensively use theories, ideals, technology, and other alternative foundations for design. Design exploration often seeks to test ideas and to ask "What if?" questions through designbut also aims to provoke, criticize, and experiment to reveal alternatives to the expected and traditional, aspiring to transcend accepted paradigms and bring matters to a head.

These projects are typically self-initiated. Rather than commercial objectives, design explorationsor 'critical design'-use design to critically comment on the relationship between technology and society, business, particular user groups, and science. Here, design is used to indicate the possible, desirable, ideal, or what is simply different from a mainstream view. The expression is often societal. Design exploration is thus a way to comment on a societal or cultural phenomena by bringing forth artifacts that in themselves, typically without the need of overhead explanations, make statements, offer arguments, or in other ways contribute to ongoing societal discussions or shed light on certain circumstances or events. The design methods applied in this area tend to be dialectical and interpretive and are often influenced by or entirely driven by a hypothesis or by theory. Especially in the extreme form of design exploration, critical design, the design researcher knowingly aims at stating a subjective standpoint or a design direction he or she sees as desirable.

Overall, this form of design research is strongly shaped by the ambition to explore new solutions, new directions, new technology, and new usage, to broaden the overall design space or to rock the boat, without necessarily trying to solve existing and well-defined problems.

\subsection{Research as Design Studies}

Design Studies is the activity area that most closely resembles traditional academic research disciplines. Here, the goal is to build foundational cumulative knowledge step-by-step and foster an intellectual tradition within the discipline. This typically involves the design researcher in analytic work, in taking part in and contributing to ongoing discussions about design theory, design methodology, design education, design tools and techniques, etc.

A common misunderstanding, which is unfortunately still being nurtured in certain quarters of design research, is to assume a close relationship between rigor and quantitative research. However, rigor does not necessarily increase with the use of complex statistical methods. Nor does carefully controlled experiments in a lab setting necessarily improve rigor. In a similar vein, qualitative approaches cannot per se 
be regarded less rigorous. The quality of design studies depends not simply on the methods or research techniques used but on the systematic nature and the clarity and transparency with which design researchers are able to put forward and support their claims. As with any form of research, the quality is improved if claims are coherent, how complete their chains of arguments are, how well it builds on earlier research, etc. Design studies, as defined here, is also typically valued in relation to the level of generalizability of the approach and result. Yet, given that design studies is the design research form that mostly overlap with what is traditionally seen as the scientific approach, it is also the least controversial and the form that easiest can conform to traditional definitions of rigor and relevance.

\section{BACK TO RIGOR AND RELEVANCE}

This brief expose of the design research triangle shows that each of the three forms of research has its own approach, outcomes, methods, and internal logic. Rather, when it comes to the question of what constitutes rigorous and relevant research in interaction design, we suggest that the most important difference between the three research forms is that they have different purposes. The purpose of each approach determines what methods and techniques are useful. For instance, in the design practice approach it is all about changing the present state of a situation to a preferred one, it means that methods and techniques for studying and creating a clear understanding of the particular situation become crucial. At the same time, since this approach is all about creating a design that works and changes a particular situation into an anticipated preferred situation, the approach does not really have any predetermined consideration of what constitutes rigor in the process. If the final design makes sense and is useful, that is, if the design is relevant, then rigor is not even an issue.

The three forms of research do not randomly advocate certain research methods, techniques, or tools, instead they are a consequence of years of trial and error, practice, and experience, through and by which appropriate methods have emerged. The methods that have survived have been and are continuously tested against the purpose of the approach and they have thus proven over time to deliver the kind of results looked for in a way that makes sense. We therefore make the argument that the only way to discuss and examine rigor and relevance for interaction design research is to do it in relation to the three forms of research and to their particular purposes. In establishing rigor in design exploration, an important criterion is to what extent the design researcher is able to continue to 'problem set' rather than 'problem solve'. The process of design exploration should open up a critical and creative approach that challenges mainstream assumptions in design, such as the consumer perspective, technology as tools, usability, etc. This means that rigor can only be measured in relation to how well the approach does open up a design space and less how that is done. Another important aspect of rigor in design exploration is the availability of the research. Are the results presented in such a way that, first, people are able to experience them in the way intended, such as in exhibitions, museums, galleries, on-line shows, etc. Second, are the results presented or shown in such a way that they are possible to critique?

When it comes to relevance, it is however quite difficult to examine whether a design practice project is relevant since the underlying purpose is to produce something relevant to a client and user. The notion of relevance is so intrinsic to this form of research that it becomes inescapable and at the same time almost invisible. At the same time, it is clear the relevance of a piece of techno-critical art cannot be assessed using the same criteria as those that establish relevance in design practice. Here, rather than clients, markets, and organizations, relevance is tied to the impact the results will and can have on society in a more general sense. The notion of relevance becomes much more complex, partly because there is always some uncertainty about the purpose of the research. The notion of 'exploring a design space' might sound clear, but what does it mean, exactly? How, for what purposes, and for whom is it explored?

An important but often overlooked aspect of rigor in design studies is that about being scholarly in one's approach; in presenting arguments, in caring for, knowing, and acknowledging existing literature and knowledge in the area, in choosing suitable methods and analysis techniques, and so on.

These examples of how rigor and relevance constitute themselves within in each research form show that it is not possible to apply a single overarching understanding of rigor and relevance in interaction design research.

\section{DISCUSSION AND CONCLUSIONS}

The overall argument in this paper is that research and particularly interaction design research can be done in different ways and for different purposes, as described above in the design research triangle. We have also made the case that each form of research must be examined in its own right and the notions of rigor and relevance for each of them 
have to be based on a firm understanding of the particular purpose of each approach.

We would argue that this is not done consistently in our field today. This sometimes leads to misunderstandings, confusion, and mistakes when design research papers and articles are reviewed, assessed, and evaluated. We argue that reviewers often come to apply the wrong notions of rigor and relevance to a particular research effort by not taking into consideration what form of research it is. For instance, relevance is often framed as a requirement for 'implications for design' even though that interpretation of relevance is not applicable for that particular form of research. Furthermore, relevance does not mean, necessarily, that everything explored should be of immediate use and applicability. Sometimes research that addresses internal research problems is useful and very relevant for advancing knowledge compared to for instance replication of similar types of research with close connection with practice. In cases like these, relevance has to be seen and evaluated using a longer time frame.

In this paper, we have discussed various aspects of rigor and relevance in interaction design research. However, we have not yet discussed one aspect that we think makes interaction design research unique, which is the ability to move quite freely inbetween the different activity areas of design practice, design exploration, and design studies. We believe that doing so is an important part of design research, which influences both rigor and relevance. It may in fact be a key characteristic of interaction design research that the different activity areas lead to multistable relationships between rigor and relevance. It is hence vital for interaction design researchers to understand where in the model they are and how they move in it to be able to establish criteria of rigor and relevance in their research.

With the diversity of research approaches in interaction design research, we argue that some notions of rigor and relevance rigor are important to the field in that they enforce and further develop the yardsticks of the different research traditions that are hidden in this diversity. Non-rigorous research in interaction design may otherwise be impossible to separate from consultancy work, journalism, or simply matters of opinion. Such yardsticks make up the standards for the field in terms of what are acceptable levels of quality for work being put forward as interaction design research.

As we mentioned in the introduction, this means that the burden of exploring as well as imposing notions of rigor and relevance falls on the field itself. Our main contribution is to point at the need to do this in relation to the specific nature of interaction design research and that our yardsticks thus cannot simply be borrowed from other fields. Researchers and educators in the field and particularly in institutions not traditionally grounded in the practice of academic research have to take on this challenge in a serious way.

\section{REFERENCES}

Buchanan, R. (1996) Wicked Problems in Design Thinking. In The Idea of Design. MIT Press, 3-20.

Cross, N. (1999) Design Research: A Disciplined Conversation, Design Issues 15, 2, 5-10.

Fallman, D. (2008) The Interaction Design Research Triangle of Design Practice, Design Exploration, and Design Studies. Design Issues, 24, 3, 4-18

Keen, P. (1991) Relevance and Rigor in Information Systems Research. In Information systems research: Contemporary approaches and emergent traditions, 27-49, Elsevier.

Lee, A. S. \& Baskerville, R. L. (2003) Generalizing Generalizability in Information Systems Research. Information Systems Research, 14, 221-243.

Mason, J. (1996) Qualitative Researching. Sage Publications, London, UK.

Robey, D. \& Markus, M. (1998) Beyond Rigor and Relevance: Producing Consumable Research about Information Systems. Information Resources Management Journal, 11, 1, 7-15

Rogers, Y. (2004) New Theoretical Approaches for Human-Computer Interaction. Annual Review of Information, Science and Technology, 38, 87-143

Roth, S. (1999) The State of Design Research. Design Issues 15, 2 (Summer 1999), 18-26.

Stolterman, E. (2008) The nature of design practice and implications for interaction design research. International Journal of Design, 2, 1

Stolterman, E. \& Wiberg, M. (forthcoming 2010) Concept-driven Interaction Design Research, The $\mathrm{HCl}$ Journal.

Yin, R.K. (1994) Case Study Research: Design and Methods. Sage.

Zimmerman, J., Forlizzi, J., \& Evenson, S. (2007). Research Through Design as a Method for Interaction Design Research in $\mathrm{HCl}$. CHI 2007. New York, NY: ACM Press, 493-502. 